\title{
Predictive Assessment of Quality of Mineral Aggregates Disintegration
}

\author{
S. Shevchenko, R. Brodskaya ${ }^{(凶)}$, I. Bilskaya, Yu. Kobzeva, \\ and V. Lyahnitskaya \\ Karpinsky Russian Geological Research Institute, St. Petersburg, Russia \\ Rimma_Brodskaya@vsegei.ru
}

\begin{abstract}
The paper is supposed to discuss one of the most important technological properties of mineral aggregates: their ability to disintegrate, to be destroyed for the subsequent redistribution and extraction of a useful component. The strength of a mineral aggregate is determined by the strength of accretion of composing individuals and depends on many factors, including the energy (kinetics) of formation conditions and the subsequent transformation conditions. The energy of the boundary of accretion of mineral grains in aggregate cannot be directly measured since currently, there are no reliable and proved methods for measuring the surface energy of solids. It is only possible to calculate quantity, proportional to the surface energy of the fusion of mineral grains - this is the calculation of the atomic density of the surface, parallel to the boundary of accretion of a pair of mineral grains. The methodology and calculation of this characteristic have been developed and published. The use and obtaining of results are possible at the stage of preliminary mineralogical study by methods of geometrical analysis of the structure of the mineral aggregate. The effectiveness of the proposed method is determined by the knowledge of the quality and features of destruction of the aggregates using different physical methods of disintegration: traditional mechanical, ultrasonic, electro-pulse, electro-hydraulic.
\end{abstract}

Keywords: Technological properties $\cdot$ Mineral aggregate $\cdot$

Aggregate disintegration - Repartition - Strength - Accretion boundaries -

Energy of boundaries

\section{Introduction}

The mineral aggregate is considered as a structured system consisting of mineral individuals and the accretion boundaries between them. The formation of the aggregate results from cooperative thermodynamic processes of synthesis of substances, corresponding to the composition of minerals, as well as the crystallization of mineral individuals and the formation of crystal-grain boundaries, their aggregation. The aggregation or accretion of mineral individuals with each other is carried out along the forming boundaries of accretion. The process of aggregation of grains in the mineral aggregate may somewhat lag behind the synthesis and crystallization of individuals, but their parallel development is also possible. The accretion boundaries do not always 
correspond to the boundaries of the crystallization of individuals, since they are formed in a different energy situation.

\section{Methods and Approaches}

The accretion boundaries of mineral individuals represent a certain transition zone between crystal lattices of accreted grains. The orientation of the accretion boundaries with respect to the symmetry of crystalline lattices of mineral grains depends on the local energy potentials during the formation and transformation of the mineral individuals and the aggregate. Local thermodynamic potentials of the forming mineral system directly affect the safety of an individual through the energy of its boundary, the choice of the orientation of the boundary relative to the symmetry of its crystal lattice and the orientation of the accretion boundary with the nearest stable grain. Thus, a compromise boundary is established for the accretion of each pair of mineral individuals, which is stable in a specific range of thermodynamic forces and flows.

During evaluation of sequence and quality of opening of the accretions, it is necessary to take into account the distribution of mineral individuals in the aggregate which possess a very perfect cleavage.

It is possible to assess the degree and quality of opening of the accretions at the stage of preliminary mineralogical analysis when studying the structure of the mineral aggregate in thin sections using geometric analysis methods.

\section{Results and Discussion}

The boundaries of mineral individuals, as well as the accretion boundaries, possess a certain stability reserve, ensuring their functioning in a certain range of thermodynamic and mechanical parameters. The stability margin of the boundaries is the wider, the higher the kinetics of the cooperative processes that form them, their genesis. The accretion boundaries of mineral grains are the more stable in the field of destructive forces, the more surface energy they accumulate.

The stability of the accretion boundary of mineral grains depends, in addition to the above said, on the degree of its balance by the value of energy saturation of each of the mineral grains in the accretion region.

The open boundaries of the mineral grains of accretions after relaxation of the crystal lattice, as well as remaining unopened accretions, have different elemental composition, amount of energy, and different floatability.

\section{Conclusions}

The accretion boundaries determine the grain system as a mineral aggregate and determine its stability, as both the strength of the accretions of mineral grains, and the strength of the mineral aggregate as a whole. 
The strength of mineral aggregates is understood as resistance to destructive forces on the system of mineral grains forming the aggregate due to the presence of their "accretion" surfaces.

The accretion boundaries of the mineral grains can be balanced or unbalanced by value of energy of accretion surfaces of individuals.

The accretion of grains on surfaces, which atomic density or crystallographic indices correspond to the edges of habit forms, will collapse faster and earlier by the same destructive force, than the accretion of energy-intensive boundaries with a low atomic density and high crystallographic indices.

\section{References}

Brodskaya RL, Makagonov EP (1990) Determination of the spatial orientation of crystalline individuals in microstructural analysis and prospects for its use in stereometric petrography. Notes All-Union Mineral Soc 119(4):84-93

Brodskaya RL, Bil'skaya IV, Lyakhnitskaya VD, Markovsky BA, Sidorov EG (2007) Boundaries of accretions between mineral individuals in an aggregate. Geol Ore Deposits 49(8):669-680

Open Access This chapter is licensed under the terms of the Creative Commons Attribution 4.0 International License (http://creativecommons.org/licenses/by/4.0/), which permits use, sharing, adaptation, distribution and reproduction in any medium or format, as long as you give appropriate credit to the original author(s) and the source, provide a link to the Creative Commons license and indicate if changes were made.

The images or other third party material in this chapter are included in the chapter's Creative Commons license, unless indicated otherwise in a credit line to the material. If material is not included in the chapter's Creative Commons license and your intended use is not permitted by statutory regulation or exceeds the permitted use, you will need to obtain permission directly from the copyright holder.

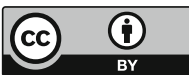

